\title{
Aplicación de una ley matemática exponencial a la dinámica cardíaca en 16 horas: estudio realizado con 250 pacientes
}

\author{
Application of an exponential mathematical law to cardiac dynamics in 16 hours: a study \\ of 250 patients
}

\section{Signed E. Prieto-Bohórquez ${ }^{1}$, Javier Rodríguez-Velásquez ${ }^{1 *}$, Catalina Correa-Herrera ${ }^{1}$, Luis M. Beltrán², Luis M. Agudelo-Otalora ${ }^{3}$, Carlos Trejos-Ramírez ${ }^{4}$ y Clímaco Pérez-Molina ${ }^{5}$}

${ }^{1}$ Grupo Insight, Hospital Universitario Nacional de Colombia, Bogotá; ${ }^{2}$ Grupo Control y Automatización de Procesos (CAPSAB), Universidad de la Sabana, Chía; ${ }^{3}$ Grupo Human Centered Design (HCD), Universidad de la Sabana, Chía; ${ }^{4}$ Departamento de Ginecología y Obstetricia, Universidad de la Sabana, Chía; ${ }^{5}$ Departamento de Cardiología, Clínica de la Sabana, Chía. Colombia

\section{Resumen}

Introducción: Los sistemas dinámicos y la geometría fractal han sido el sustrato para el advenimiento de una ley matemática aplicada al diagnóstico de la dinámica cardíaca en 21 horas. Objetivo: Confirmar la aplicabilidad clínica de la ley matemática exponencial en 16 horas a partir de un estudio de concordancia diagnóstica frente a la norma de referencia. Materiales y método: Se realizó un estudio con 250 registros electrocardiográficos continuos y ambulatorios; 50 pertenecían a pacientes normales y 200 a pacientes con diversas enfermedades cardíacas. Se simuló la secuencia de frecuencias cardíacas y se construyeron los atractores correspondientes. Se calculó la dimensión fractal y la ocupación del atractor en el espacio generalizado de box-counting. Por último, se estableció el diagnóstico fisicomatemático en 16 y 21 horas y se efectuó la validación estadística. Resultados: Los espacios de ocupación para normalidad en la rejilla pequeña se encontraron entre 205 y 372, y entre 56 y 201 para dinámicas patológicas, lo cual permitió evidenciar la capacidad del método para diferenciar normalidad de enfermedad a través de la ocupación espacial de los atractores con base en la ley matemática en 16 horas. Se hallaron valores de sensibilidad y especificidad del 100\% y un coeficiente kappa del orden de 1, luego de comparar el diagnóstico fisicomatemático frente a la norma de referencia. Conclusión: La ley matemática exponencial en 16 horas demostró su utilidad como herramienta de ayuda diagnóstica y predictiva, lo cual permitió diferenciar normalidad y estados evolutivos hacia enfermedad y agudización.

Palabras clave: Enfermedad cardiovascular. Sistemas dinámicos. Diagnóstico. Holter.

\begin{abstract}
Introduction: Dynamic systems and fractal geometry have been the substrate for the rising of a mathematical law applied to the diagnosis of cardiac dynamics in 21 hours. Objective: To confirm the clinical applicability of the exponential mathematical law in 16 hours, with a study of diagnostic agreement against the Gold Standard. Materials and method: It was made a study with 250 ambulatory and continuous electrocardiographic recordings, 50 belonged to normal patients and 200 to
\end{abstract}

\section{Correspondencia:}

*Javier Rodríguez-Velásquez

E-mail: grupoinsight2025@gmail.com
Disponible en internet: 29-07-2021 Rev Colomb Cardiol. 2021;28(3):231-238 www.rccardiologia.com 0120-5633 / @ 2020 Sociedad Colombiana de Cardiología y Cirugía Cardiovascular. Publicado por Permanyer. Este es un artículo open access bajo la licencia CC BY-NC-ND (http://creativecommons.org/licenses/by-nc-nd/4.0/). 
patients with various cardiac pathologies. The sequence of heart rates was simulated, and attractors were constructed. It was calculated the fractal dimension of the attractor and its occupation in the generalized Box-Counting space. Finally, it was determined the physical-mathematical diagnostic in 16 and 21 hours, and statistical validation was performed. Results: The occupation spaces in the small grid were between 205 and 372 for normality, and between 56 and 201 for pathologic dynamics, which demonstrated the ability of the method to differentiate normal condition from sickness, through spatial occupation of attractors according to mathematical law in 16 hours. There were obtained values of sensitivity and specificity of $100 \%$ and Kappa coefficient was 1, after comparing the physic-mathematical analysis against the Gold Standard. Conclusion: The exponential mathematical law in 16 hours proved its utility as diagnostic and predictive tool support, allowing to differentiate normal, developmental stages to disease and exacerbation.

Key words: Cardiovascular disease. Dynamical systems. Diagnostics. Holter.

\section{Introducción}

La teoría de los sistemas dinámicos permite analizar el estado y la evolución de los sistemas; estos últimos se conciben como conjuntos de elementos en interacción y pueden ser predecibles o impredecibles. Con el fin de alcanzar una mayor comprensión de dichos conjuntos de elementos se han desarrollado procedimientos fisicomatemáticos que permiten analizar variaciones en el tiempo de las distintas variables que los componen ${ }^{1}$. Los espacios de fases constituyen espacios geométricos en los cuales se realizan representaciones gráficas de los sistemas y su evolución. Las trayectorias identificadas de las dinámicas permiten dilucidar si éstas son predecibles o impredecibles ${ }^{2} y$, en función de ello, se establecen diferentes tipos de atractores. Éstos pueden ser puntuales o cíclicos, para sistemas predecibles, o caóticos, si son impredecibles, como en el caso de la dinámica cardíaca. Dada la irregularidad de los atractores caóticos, su cuantificación se realiza a la luz de la geometría fractal que desarrolló Benoît Mandelbrot, gracias a la cual es posible realizar mediciones de objetos irregulares ${ }^{3,4}$, tras considerar que su medición no es posible mediante la geometría euclidiana.

Las enfermedades cardiovasculares se han consolidado como uno de los principales desafíos del siglo XXI en el ámbito de la salud. Según la Organización Mundial de la Salud, se calcula que la cantidad de muertes relacionadas con esta causa aumentará hasta un valor de 25 millones en el año $2030^{5}$, en cuyo caso las naciones en vías de desarrollo serían las más afectadas, dado que el $80 \%$ de dichas enfermedades ocurre en estas regiones ${ }^{6}$.

Si se considera la problemática actual, es necesario desarrollar nuevos métodos de detección oportuna de enfermedades cardíacas y mejorar los existentes para reducir la morbimortalidad vinculada con esta causa. El registro electrocardiográfico ambulatorio, también conocido como Holter, es uno de los estudios de mayor relevancia clínica para identificar alteraciones del ritmo cardíaco ${ }^{7}$. En las unidades de cuidados intensivos se emplean monitores de registro electrocardiográfico continuo en el seguimiento de pacientes críticos.

El intervalo RR y su correspondiente análisis permite el establecimiento de algunas diferenciaciones entre estados normales y patológicos ${ }^{7-9}$; sin embargo, aún se evidencian limitaciones en cuanto a su aplicación clínica. El análisis de este parámetro se ha realizado a la luz de la perspectiva homeostática tradicional preestablecida en la fisiología, según la cual se plantea que la normalidad se relaciona con estados regulares ${ }^{10}$. Distintos estudios plantean que esta perspectiva posee limitaciones en la comprensión del comportamiento de la dinámica cardíaca y se han presentado nuevas vías de análisis con base en teorías y métodos fisicomatemáticos, entre los que figuran los sistemas dinámicos, la teoría del caos y la geometría fractal11-14.

Goldberger, et al. han realizado estudios en el marco de los sistemas dinámicos que han permitido el establecimiento de una nueva concepción de normalidad y enfermedad, distinta de la establecida desde la fisiología convencional. Según esta nueva perspectiva, no sólo las dinámicas en extremo aleatorias se vinculan con enfermedad; también las dinámicas muy regulares o periódicas se relacionan con estados patológicos, mientras que la normalidad se halla en el intervalo encontrado entre estos dos patrones comportamentales extremos ${ }^{15}$. El advenimiento de esta perspectiva dio origen a trabajos como el de Huikuri, et al., en el que se establecieron nuevos índices de mortalidad con fines predictivos en pacientes que cursaban con síndrome coronario agudo tipo infarto agudo de miocardio y fracción de expulsión ventricular gravemente disminuida, lo que supera algunas limitaciones de parámetros convencionales ${ }^{16}$.

Desde la perspectiva fisicomatemática y al tomar como epicentro los sistemas dinámicos y la geometría 
fractal, Rodríguez, et al. ${ }^{17}$ desarrollaron una ley matemática exponencial aplicada a los sistemas caóticos cardíacos, a través de la cual se diagnostica y cuantifica el nivel de gravedad para cada dinámica, luego de establecer diferencias entre normalidad, enfermedad y evolución hacia estados patológicos. De manera adicional, con esta metodología se estableció la totalidad de las dinámicas posibles, según cada estado. La capacidad diagnóstica y predictiva de esta ley se ha corroborado en diversos estudios ulteriores, incluido un estudio ciego desarrollado con 115 casos $^{18}$, con registro de valores de sensibilidad y especificidad del orden de $100 \%$, así como un coeficiente kappa con un valor de 1. También se ha aplicado a pacientes que cursan con arritmias ${ }^{19,20}$, lo cual permite evidenciar la capacidad del método en la detección de alteraciones leves subdiagnosticadas y en la medición del grado de evolución de la arritmia.

En fecha reciente se desarrolló un trabajo de investigación en el que se reveló la capacidad de esta ley matemática para diagnosticar, de manera precisa, incluso en períodos de 18 horas, al establecer la importancia de su implementación clínica (en evaluación para publicación). El objetivo de este trabajo es corroborar la capacidad diagnóstica de esta ley con una reducción del tiempo de evaluación a 16 horas, a través de su aplicación a 250 casos y su comparación frente a la norma de referencia, en el marco de un estudio ciego.

\section{Materiales y método}

\section{Definiciones}

1. Espacio de fases: espacio de dos o más dimensiones que permite representar geométricamente el cambio de un sistema en el tiempo mediante una gráfica de pares ordenados, o pareja de elementos matemáticos que corresponden a una variable, que en este caso se relaciona con los valores de la frecuencia cardíaca consecutiva.

2. Dimensión fractal de box-counting: es una expresión matemática que se refiere a la complejidad de una estructura irregular medida por el método de box-counting, que se define por la ecuación 1 , y consiste en la superposición de dos rejillas sobre el objeto para cuantificar su ocupación fractal.

3. Ecuación de box-counting simplificada: surge como una simplificación de la ecuación 1 , al considerar para el análisis de la cuantificación del atractor cardíaco dos cuadrículas que tienen una proporción de
$1 / 2$ en sus dimensiones, es decir, una de las rejillas posee el doble de longitud en sus lados, con lo que se obtiene la ecuación 2.

4. Ley exponencial de la dinámica caótica cardíaca ${ }^{17-21}$ : se obtiene al despejar la ecuación 2 en términos de Kp, para obtener la ecuación 3, en la cual se deduce la relación entre el número de espacios ocupados de la dimensión fractal. Además, la forma de la ecuación cambia de una logarítmica a una exponencial.

\section{Población}

A partir de una base de datos del grupo Insight de registros continuos y ambulatorios de electrocardiografía recopilada entre el 2016 y el 2017 de investigaciones previas en el área de cardiología, con aplicación de teorías físicas y matemáticas con la Clínica del Country en Bogotá, se verificó que estos registros indicaran las frecuencias cardíacas mínimas y máximas al igual que la cantidad de latidos durante cada hora por al menos 21 horas consecutivas para ser incluidos. Se tomaron 250 registros de electrocardiografía provenientes de individuos mayores de 21 años y que tuvieran un diagnóstico final emitido por un profesional subespecialista en cardiología o electrofisiología. De los 250 registros, 50 se diagnosticaron como normales, mientras que 200 correspondieron a casos con distintos tipos de alteraciones cardíacas.

\section{Procedimiento}

De modo inicial se ocultaron los diagnósticos clínicos emitidos de acuerdo con los parámetros clínicos convencionales de los registros electrocardiográficos. Luego, a partir de estos registros, se revisaron y tomaron los valores máximos y mínimos de las frecuencias cardíacas y el número total de latidos durante cada hora por 21 y 16 horas.

Estos valores se organizaron por hora y dispusieron en un software con el cual se generó una secuencia seudoaleatoria que concibe los valores de las frecuencias cardíacas mínimas y máximas como los límites de la secuencia y la cantidad de latidos como la cantidad de valores que la componen a través de un algoritmo equiprobable.

Con posterioridad se empleó dicha secuencia para construir un atractor caótico y se graficó la primera frecuencia cardíaca contra la siguiente y así de manera consecutiva hasta la última a lo largo del tiempo, en el correspondiente espacio de fases (ecuación 1). Después se calculó la dimensión fractal a través del método 
de box-counting (ecuación 2) mediante la superposición de las dos rejillas de 5 latidos/min (Kp) y 10 latidos/min $(\mathrm{Kg})$, y se cuantificaron los espacios ocupados por cada atractor en cada una de dichas rejillas.

$$
D=\frac{\log N\left(2^{-(K+1)}\right)-\log N\left(2^{-K}\right)}{\log 2^{k+1}-\log 2^{k}}
$$

Ecuación 1

En esta ecuación, D corresponde a la dimensión fractal y $\mathrm{N}$ es el número de cuadros ocupado por el objeto en la cuadrícula de grado de partición $\mathrm{k}$.

$$
D=\log _{2}\left[\frac{K_{p}}{K_{g}}\right]
$$

Ecuación 2

En ésta, Kp es el número de cuadros ocupados para la cuadrícula más pequeña de 5 latidos/min y $\mathrm{Kg}$ es el número de cuadros ocupados para la cuadrícula grande de 10 latidos $/ \mathrm{min}$.

De acuerdo con la ley ya desarrollada ${ }^{17-21}$, para los casos de enfermedad aguda se evidencian espacios de ocupación en $\mathrm{Kp}<73$, mientras que la normalidad presenta espacios de ocupación $\mathrm{Kp}>200$ y, por último, los estados evolutivos hacia enfermedad se encuentran en el intervalo de espacios de ocupación comprendido entre 73 y 200. Estos valores se obtienen al realizar un cálculo con la ecuación 3 e indican la ocupación que presenta el atractor cardíaco en la rejilla Kp, lo que logra establecer el diagnóstico físico y matemático para cada registro.

$$
K_{p}=2^{D} K_{g}
$$

\section{Ecuación 3}

\section{Análisis estadístico}

Se ocultaron los diagnósticos clínicos de cada uno de los registros, tanto ambulatorios como continuos. Se tomaron como norma de referencia los diagnósticos establecidos por un cardiólogo experto según parámetros convencionales y se compararon con los diagnósticos fisicomatemáticos en 16 horas. Se tomaron los casos de enfermedad aguda y normalidad para efectos del análisis estadístico, previa confirmación de la concordancia diagnóstica en los diagnósticos en 16 y 21 horas.

Se calcularon los falsos negativos, falsos positivos, verdaderos positivos $\mathrm{y}$ verdaderos negativos con una tabla de contingencia de $2 \times 2$, de tal forma que los verdaderos positivos (VP) se representaron por el número de pacientes diagnosticados clínica y matemáticamente con enfermedad aguda; los falsos positivos (FP) correspondieron al número de registros diagnosticados con enfermedad aguda por la metodología fisicomatemática, pero cuyo diagnóstico clínico fue de normalidad; los falsos negativos (FN) fueron el número de registros ambulatorios y continuos que matemáticamente se caracterizaron como normales, pero cuyo diagnóstico clínico fue de enfermedad aguda; y los verdaderos negativos (VN) se entendieron como el número de registros con diagnóstico clínico y matemático de normalidad. También se determinó la concordancia entre la norma de referencia y la metodología fisicomatemática a partir del coeficiente Kappa mediante la ecuación 4.

$$
K=\frac{C o-C a}{T o-C a} \quad \text { Ecuación } 4
$$

En esta fórmula, Co representa el número de concordancias evidenciadas, la totalidad de pacientes igualmente diagnosticados desde la metodología fisicomatemática y los parámetros clínicos convencionales; To corresponde a la totalidad de casos; Ca es la totalidad de concordancias atribuidas al azar calculadas con la ecuación 5 .

$$
C a=\left[\left(f_{1} x C_{1}\right) / T o\right]+\left[\left(f_{2} x C_{2}\right) / T o\right] \quad \text { Ecuación } 5
$$

En ésta, $\mathrm{f}_{1}$ es el número de casos con valores matemáticos correspondientes a normalidad; $c_{1}$ son los casos diagnosticados desde los parámetros clínicos como normales; $f_{2}$ son los casos evaluados matemáticamente como patológicos y $\mathrm{c}_{2}$ son los casos diagnosticados como patológicos según los protocolos clínicos convencionales; To es la totalidad de casos.

\section{Aspectos éticos}

Esta investigación cumple con los principios éticos, técnicos y científicos consignados en la declaración de Helsinki de la Asociación Médica Mundial; también se apegó a los parámetros de la resolución 8430 de 1993 del Ministerio de Salud Colombiano. Se catalogó como una investigación de riesgo mínimo, dado que en ningún momento se solicitaron pruebas diagnósticas ni se realizaron procedimientos invasivos y sólo se efectuaron cálculos físicos y matemáticos con base en informes de estudios y paraclínicos no invasivos prescritos con anterioridad, según los criterios clínicos del estudio de enfermedades cardíacas. Asimismo, la información analizada en las bases de datos se encontraba codificada para garantizar la protección de los datos y la integridad de los participantes.

\section{Resultados}

En la Tabla 1 se observa el diagnóstico desde el punto de vista clínico de algunos registros electrocardiográficos Holter y continuos. En el caso de las 
Tabla 1. Información clínica de algunos de los registros electrocardiográficos del estudio

\begin{tabular}{|c|c|}
\hline Holter no. & Diagnóstico \\
\hline 1 & Control taquicardia supraventricular \\
\hline 2 & Síncope en estudio \\
\hline 3 & Miocardiopatía hipertrófica (MCH) \\
\hline 4 & Normal \\
\hline 5 & Infarto cerebral \\
\hline 6 & Normal \\
\hline 7 & Hipertrofia septal asimétrica \\
\hline 8 & Normal \\
\hline 9 & $\begin{array}{l}\text { Extrasistolia auricular conducida infrecuente sin } \\
\text { fenómenos repetitivos } \\
\text { Extrasistolia ventricular aislada }\end{array}$ \\
\hline 10 & Fibrilación auricular \\
\hline 11 & Normal \\
\hline 12 & Normal \\
\hline 13 & Normal \\
\hline 14 & Taquicardia supraventricular \\
\hline 15 & Bloqueo de CIA \\
\hline 16 & Normal \\
\hline 17 & Arritmia \\
\hline 18 & Análisis de presíncope \\
\hline 19 & Mareo, taquicardia supraventricular paroxística \\
\hline 20 & POP, ablación \\
\hline 21 & Palpitaciones, arritmia \\
\hline 22 & Normal \\
\hline 23 & Normal \\
\hline 24 & Normal \\
\hline 25 & Taquicardia \\
\hline 26 & Normal \\
\hline 27 & Normal \\
\hline 28 & Miocardiopatía idiopática \\
\hline 29 & Normal \\
\hline 30 & $\begin{array}{l}\text { Insuficiencia cardíaca descompensada. IAM } \\
\text { CEST, síndrome cardiorrenal tipo } 1\end{array}$ \\
\hline 31 & $\begin{array}{l}\text { Bradicardia; disminución moderada de la } \\
\text { variabilidad de la frecuencia cardíaca }\end{array}$ \\
\hline
\end{tabular}

dinámicas cardíacas evaluadas en 21 horas, la dimensión fractal de los atractores normales se encontró entre 0.827 y 1.921 , mientras que la dimensión fractal de las dinámicas anormales se halló entre 0.846 y 1.823. La dimensión fractal de los atractores normales en 16 horas registró 0.825 a 1.974, en tanto que para el caso de los atractores anormales fue de 0.828 a 1.930; al llevar a cabo el comparativo entre las dimensiones fractales entre 16 y 21 horas se observa su similitud (Fig. 1). Estos valores corroboran hallazgos previos, en los que se advierte que los valores de la dimensión fractal no diferencian adecuadamente las dinámicas cardíacas, ni en 16 ni en 21 horas.

No obstante, las dinámicas normales evaluadas en 21 horas en la rejilla pequeña revelaron espacios de ocupación de 200 a 363, mientras que las anormales presentaron espacios de ocupación de 55 a 196. Las dinámicas normales evaluadas en 16 horas en la rejilla pequeña tuvieron espacios de ocupación de 205 a 372, en tanto que las anormales evaluadas en 16 horas tuvieron espacios de 56 a 201 (Tabla 2).

En cuanto a los espacios de ocupación de la rejilla $\mathrm{Kg}$, en 21 horas se encontró que las dinámicas normales tenían valores entre 57 y 182, mientras que las anormales presentaron valores de 18 a 96 . Los espacios de ocupación de las dinámicas normales en la rejilla Kg en 16 horas se hallaron entre 56 y 184, y para dinámicas anormales entre 21 y 94 (Tabla 2). Hubo concordancia en los diagnósticos matemáticos en 16 y 21 horas en el $100 \%$ de los casos y se evidenciaron valores de sensibilidad y especificidad del $100 \%$, con un coeficiente kappa de 1.

En la Figura 2 se muestran los atractores para una dinámica normal, en evolución hacia la agudización y aguda, lo que revela cómo se reduce el tamaño del atractor mientras la enfermedad se agudiza.

\section{Discusión}

Éste es el primer trabajo en el cual se confirma, a través de un estudio de concordancia diagnóstica, la capacidad de la ley caótica exponencial aplicada a la dinámica cardíaca de establecer diagnósticos mediante una diferenciación matemática de dinámicas normales, con enfermedad aguda y en evolución entre estados, luego de realizar una reducción del tiempo de evaluación a 16 horas, en el marco de un estudio ciego con 250 registros electrocardiográficos continuos y ambulatorios. Se obtuvieron valores de sensibilidad y especificidad del orden del $100 \%$ y un coeficiente kappa con valor de 1 al comparar la metodología fisicomatemática desarrollada frente al diagnóstico convencional, tomado como norma de 


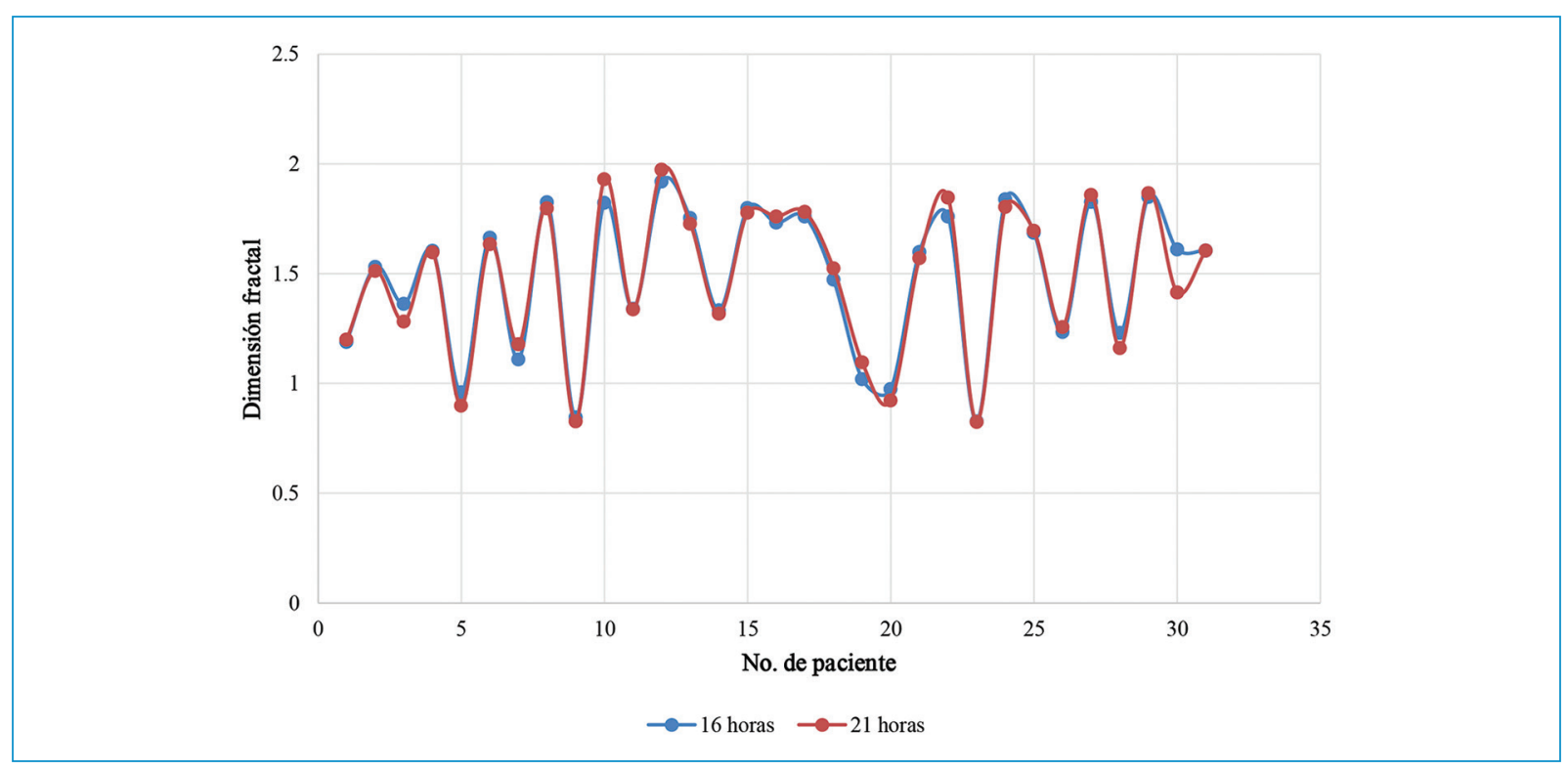

Figura 1. Comparativo entre las dimensiones fractales entre 16 y 21 horas para los pacientes incluidos en la Tabla 1.

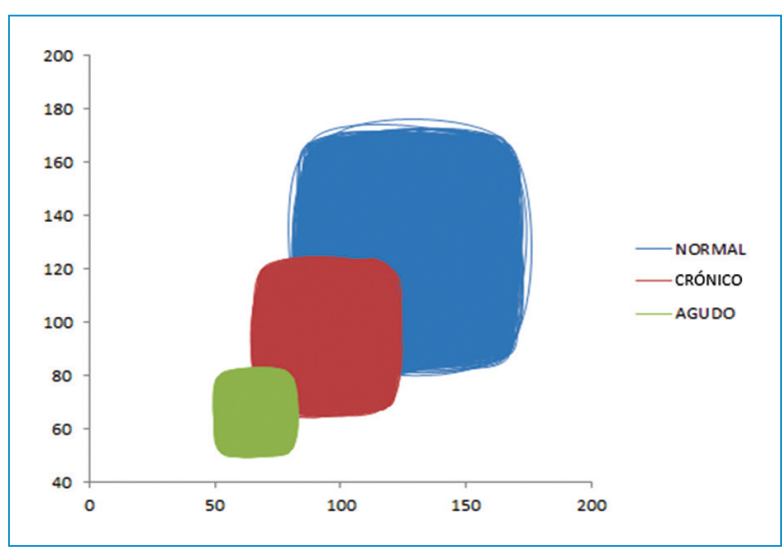

Figura 2. Atractores de la dinámica cardíaca. Ejemplos de las dinámicas normal, crónica o en evolución hacia la agudización y aguda. Los espacios de ocupación de estos atractores son para el caso agudo $\mathrm{Kp} 56$ y Kg 21 (caso no. 30 de la Tabla 1), para evolución hacia agudización Kp 146 y Kg 48 (caso no. 31 de la Tabla 1) y para el normal Kp 372 y Kg 102 (caso no. 29 de la Tabla 1).

referencia. Dado que esta metodología permite la cuantificación del nivel de gravedad de las distintas enfermedades, se consolida como un método predictivo que permite la detección de casos con alteraciones leves, que pueden subdiagnosticarse con los métodos convencionales; asimismo, permite la medición del nivel de gravedad de los casos con dinámica cardíaca patológica aguda o en evolución hacia enfermedad mediante el descenso de los valores de la ocupación espacial del atractor cardíaco en el espacio generalizado de box-counting, a la luz de la evaluación con la mencionada ley matemática exponencial.

La ley matemática de los sistemas dinámicos cardíacos aplicada en este trabajo investigativo se desarrolló a partir de un razonamiento fisicomatemático teórico e inductivo que permitió, en trabajos previos, determinar la totalidad de las dinámicas cardíacas posibles. Dado el carácter objetivo y reproducible de la ley exponencial, es más sencilla su automatización para su posterior aplicación en la práctica clínica. Su capacidad diagnóstica se ha evidenciado en el análisis de la dinámica cardíaca en general ${ }^{17,18}$ y en casos de pacientes que cursan con arritmias ${ }^{19-21}$. En esta investigación se corrobora que, aun cuando se reduzca el tiempo de evaluación de la dinámica a 16 horas, se observa su aplicabilidad clínica, lo cual permitiría en el futuro un tiempo más corto de respuesta clínica en situaciones que comprometan la vida de los pacientes, con implicaciones globales.

La fisiología clásica se fundamenta en una noción puramente homeostática y orientada al equilibrio, según la cual la normalidad se relaciona con comportamientos regulares, periódicos, o ambos ${ }^{22}$, un aspecto que ha sido controvertido a la luz de los hallazgos encontrados en investigaciones que plantean nuevas perspectivas, como aquellas en las que se incorporan 
Tabla 2. Valores de los espacios de ocupación de los atractores cardíacos evaluados tanto en 16 como en 21 horas, correspondientes a los registros electrocardiográficos continuos o Holter de la Tabla 1

\begin{tabular}{|c|c|c|c|c|c|c|}
\hline \multirow[t]{2}{*}{ Holter no. } & \multicolumn{3}{|c|}{21 horas } & \multicolumn{3}{|c|}{16 horas } \\
\hline & Kp & $\mathrm{Kg}$ & DF & Kp & $\mathbf{K g}$ & DF \\
\hline 1 & 187 & 82 & 1.1893 & 192 & 83 & 1.200 \\
\hline 2 & 133 & 46 & 1.5317 & 137 & 48 & 1.5131 \\
\hline 3 & 108 & 42 & 1.3626 & 107 & 44 & 1.2820 \\
\hline 4 & 225 & 74 & 1.6043 & 224 & 74 & 1.5979 \\
\hline 5 & 70 & 36 & 0.9594 & 69 & 37 & 0.8991 \\
\hline 6 & 206 & 65 & 1.6641 & 208 & 67 & 1.6344 \\
\hline 7 & 82 & 38 & 1.1096 & 86 & 38 & 1.1783 \\
\hline 8 & 287 & 81 & 1.8251 & 285 & 82 & 1.7973 \\
\hline 9 & 160 & 89 & 0.8462 & 158 & 89 & 0.8280 \\
\hline 10 & 138 & 39 & 1.8231 & 141 & 37 & 1.9301 \\
\hline 11 & 319 & 126 & 1.3401 & 316 & 125 & 1.3380 \\
\hline 12 & 216 & 57 & 1.920 & 220 & 56 & 1.9740 \\
\hline 13 & 317 & 94 & 1.7538 & 318 & 96 & 1.7279 \\
\hline 14 & 174 & 69 & 1.3344 & 177 & 71 & 1.3179 \\
\hline 15 & 167 & 48 & 1.7987 & 168 & 49 & 1.7777 \\
\hline 16 & 246 & 74 & 1.7330 & 244 & 72 & 1.7608 \\
\hline 17 & 167 & 49 & 1.760 & 165 & 48 & 1.7814 \\
\hline 18 & 186 & 67 & 1.4731 & 187 & 65 & 1.5245 \\
\hline 19 & 196 & 96 & 1.020 & 201 & 94 & 1.0965 \\
\hline 20 & 167 & 85 & 0.9743 & 165 & 87 & 0.9234 \\
\hline 21 & 100 & 33 & 1.5995 & 101 & 34 & 1.5707 \\
\hline 22 & 200 & 59 & 1.7612 & 205 & 57 & 1.8466 \\
\hline 23 & 323 & 182 & 0.8276 & 326 & 184 & 0.8252 \\
\hline 24 & 297 & 83 & 1.8393 & 297 & 85 & 1.8049 \\
\hline 25 & 132 & 41 & 1.6868 & 136 & 42 & 1.6952 \\
\hline 26 & 313 & 133 & 1.2347 & 313 & 131 & 1.2566 \\
\hline 27 & 298 & 84 & 1.8269 & 301 & 83 & 1.8586 \\
\hline 28 & 183 & 78 & 1.2303 & 179 & 80 & 1.1619 \\
\hline 29 & 363 & 100 & 1.850 & 372 & 102 & 1.8667 \\
\hline 30 & 55 & 18 & 1.6114 & 56 & 21 & 1.4150 \\
\hline 31 & 140 & 46 & 1.6057 & 146 & 48 & 1.6049 \\
\hline
\end{tabular}

Kp: valores de la rejilla pequeña; Kg: valores de la rejilla grande; DF: dimensión fractal.

teorías y conceptos como los sistemas dinámicos en el estudio de fenómenos, entre los que figura la dinámica cardíaca ${ }^{15}$. Los hallazgos encontrados han hecho posible el establecimiento de nuevas vías de investigación con el fin de mejorar los resultados logrados mediante el análisis de la variabilidad de la frecuencia cardíaca, que sólo se habían desarrollado desde la perspectiva homeostática tradicional y daban prelación a la realización de medidas estadísticas que muestran comportamientos globales y evalúan parámetros de la dinámica respecto de promedios $u$ otras mediciones, lo cual aún posee algunas limitaciones en casos particulares. Este tipo de investigaciones ha permitido el logro de resultados parciales y ha identificado disminuciones de la variabilidad en relación con anormalidades clínicas específicas, como el síndrome coronario agudo tipo infarto agudo de miocardio ${ }^{23}$. De acuerdo con los nuevos enfoques, la evaluación de la autoorganización del sistema en función de la ley matemática aplicada en esta investigación establece nuevos elementos para la predicción de la progresión hacia enfermedad o estados de agudización.

De esta manera, esta metodología y su aplicación permitirían a los especialistas en cardiología y electrofisiología diagnosticar dinámicas cardíacas y su evolución a lo largo del tiempo en contextos agudos y ambulatorios, al confirmar la idoneidad de los tratamientos establecidos o incluso como herramienta de estratificación del riesgo cardíaco, independiente de escalas clínicas y consideraciones epidemiológicas poblacionales. Sin embargo, su utilidad en la práctica clínica se reflejará al final en la automatización mediante software.

La ley matemática empleada se fundamenta en la línea de razonamiento acausal proveniente de la física teórica moderna. Así como se han logrado predicciones en aspectos fundamentales de la historia de la ciencia al margen de relaciones causales basadas en la ley del caos ${ }^{1,13,24}$, la mecánica cuántica ${ }^{25}$ y la mecánica estadística ${ }^{26,27}$, el diagnóstico de la dinámica cardíaca se establece a partir de dicha línea de razonamiento independiente de consideraciones como causas, género, edad, factores de riesgo adjuntos $u$ otras consideraciones demográficas o estadísticas; esto hace posible establecer mediciones objetivas aplicables a casos particulares.

Los lineamientos de la perspectiva fisicomatemática acausal han permitido establecer nuevos diagnósticos y predicciones de la dinámica cardíaca. Tal es el caso de la aplicación de la ley de Zipf-Mandelbrot ${ }^{28,29}$ y de teorías como la probabilidad y la entropía ${ }^{30}$. En otras áreas del ámbito médico se han determinado diagnósticos del proceso de alteración celular cervicouterina ${ }^{31}$ de los eritrocitos ${ }^{32}$. De igual modo, esta perspectiva ha permitido realizar estudios en aspectos como el proceso de reestenosis arterial ${ }^{33}$ y se han establecido predicciones en aspectos de relevancia en salud pública, como los brotes de malaria en 820 municipios 
colombianos $^{34}$, además de efectuar predicciones del número de linfocitos TCD4 en pacientes seropositivos con parámetros del hemograma ${ }^{35}$ y en la unión peptídica al HLA clase $\|^{36}$. También se han desarrollado predicciones de mortalidad en pacientes de la unidad de cuidados intensivos, tomando como base la teoría de los sistemas dinámicos y la teoría de conjuntos ${ }^{37}$.

\section{Agradecimientos}

Los autores agradecen a las directivas de la Universidad de la Sabana, en especial a la Dirección General de Investigación, dado que este trabajo es parte de los resultados alcanzados para el proyecto MED 203-2015, financiado por la Universidad de La Sabana.

Los autores agradecen al Hospital Universitario Nacional de Colombia por su apoyo a sus investigaciones.

\section{Conflictos de intereses}

Los autores del manuscrito no tienen conflicto de intereses.

\section{Responsabilidades éticas}

Protección de personas y animales. Los autores declaran que para esta investigación no se han realizado experimentos en seres humanos ni en animales.

Confidencialidad de los datos. Los autores declaran que en este artículo no aparecen datos de pacientes.

Derecho a la privacidad y consentimiento informado. Los autores declaran que en este artículo no aparecen datos de pacientes.

\section{Bibliografía}

1. Devaney R. A first course in chaotic dynamical systems theory and experiments. Reading Mass: Addison- Wesley, 1992.

2. Peitgen $H$, Jurgens $H$, Saupe D. Chaos and fractals; new frontiers of science. New York: Springer, 1992.

3. Mandelbrot B. ¿Cuánto mide la costa de Bretaña? In: Los objetos fractales. $3^{a}$. ed. Barcelona: Tusquets, 1988:27-50.

4. Mandelbrot B. The fractal geometry of nature. Freeman. Barcelona: Tusquets 2000:341-348.

5. Organización Mundial de la Salud. Estadísticas sanitarias mundiales 2012. [Internet]. Disponible en: http://www.who.int/gho/publications/ world_health_statistics/ES_WHS2012_Full.pdf.

6. World Health Organization. Cardiovascular Disease. 2014. [Internet] [actualizado 2014; citado enero de 2013]. Disponible en: http://www.who. int/cardiovascular_diseases/en

7. Pineda M, Matiz H, Rozo R. Enfermedad coronaria. Bogotá: Editorial Kimpres, 2002.

8. Barron H, Viskin S. Autonomic markers and prediction of cardiac death after myocardial infarction. Lancet. 1998;351:461-2.

9. Wolf M, Varigos G, Hunt D, Sluman J. Sinus arrhythmia in acute myocardial infarction. Med J Aus. 1978;2:52-3.
10. Goldberger A, Rigney DR, West B. Caos y fractales en la fisiología humana. Investigación y Ciencia. 1990;163:32-8.

11. Walleczek J. Nonlinear dynamics, self-organization, and biomedicine. Cambridge, U.K: Cambridge University Press, 1999.

12. Goldberger A. Heartbeats, hormones, and Health - Is variability the spice of life? American Journal of Respiratory and Critical Care Medicine. 2001;163(6):1289-90.

13. Lavenda B. El movimiento browniano. Investigación y Ciencia. 1990;28-37.

14. Peitgen $H$, Jurgens $H$, Saupe $D$. Chaos and fractals; new frontiers of science. New York: Springer, 1992.

15. Goldberger A, Amaral L, Hausdorff JM, Ivanov P, Peng CH, Stanley HE. Fractal dynamics in physiology: alterations with disease and aging. PNAS. 2002;99:2466-72.

16. Huikuri HV, Mäkikallio T, Peng CK, Goldberger A, Hintze U, Moller M. Fractal correlation properties of R-R interval dynamics and mortality in patients with depressed left ventricular function after and acute myocardial infarction. Circulation. 2000;101:47-53.

17. Rodríguez J. Mathematical law of chaotic cardiac dynamic: predictions of clinic application. J Med Med Sci. 2011;2(8):1050-9.

18. Rodríguez J, Correa C, Melo M, Domínguez, D, Prieto S, Cardona DM, et al. Chaotic cardiac law: developing predictions of clinical application. J Med Med Sci. 2013;4(2):79-84.

19. Rodríguez J, Prieto S, Domínguez D, Correa C, Melo M, Pardo J, et al. Application of the chaotic power law to cardiac dynamics in patients with arrhythmias. Revista de la Facultad de Medicina. 2014;62(4):539-46.

20. Rodríguez J, Prieto S, Correa C, Soracipa Y, Cardona DM, Prieto I, et al. Ley matemática para evaluación de la dinámica cardíaca: aplicación en el diagnóstico de arritmias. Rev Cienc Salud. 2015;13(3):369-81.

21. Rodríguez J, Narváez R, Prieto S, Correa C, Bernal P, Aguirre G, et al. The mathematical law of chaotic dynamics applied to cardiac arrhythmias. J Med Sci. 2013;4(7):291-300.

22. Guyton A, Hall J. Organización funcional del cuerpo humano y control del «medio interno». En: Guyton \& Hall (eds.). Tratado de fisiología médica. 12a ed. Barcelona: Elsevier, 2011:3-10.

23. Malik M, Farrell T, Cripps T, Camm AJ. Heart rate variability in relation to prognosis after myocardial infarction: selection of optimal processing techniques. Eur Heart J. 1989;10:1060-74.

24. Crutchfield J, Farmer D, Packard N, Shaw R. Caos. In: Crutchfield J Farmer D, Packard N, Shaw R. Orden y caos. Scientific American. Barcelona: Prensa Científica 1990:78-90.

25. Feynman R. Comportamiento cuántico. In: Feynman RP, Leighton RB Sands M. Física. Wilmington: Addison-Wesley Iberoamericana, 1964:371, 37-16.

26. Feynman RP, Leighton RB, Sands M. Leyes de la termodinámica. En: Feynman RP, Leighton RB, Sands M. Física. Wilmington: Addison-Wesley Iberoamericana 1964:44.1-44.19

27. Tolman R. Principles of statistical mechanics. New York: Dover Publications, 1979.

28. Rodríguez J, Prieto S, Correa C, Mendoza F, Weisz G, Soracipa M, et al. Physical mathematical evaluation of the cardiac dynamic applying the Zipf- Mandelbrot law. Journal of Modern Physics. 2015;6(13):1881-8.

29. Rodríguez J, Prieto S, Ortiz L, Bautista A, Bernal P, Avilán N. Diagnóstico matemático de la monitoría fetal aplicando la ley de Zipf-Mandelbrot. Revista de la Facultad de Medicina 2006;54(2):96-107.

30. Rodríguez J, Prieto S, Domínguez D, Melo M, Mendoza F, Correa M, et al. Mathematical-physical prediction of cardiac dynamics using the proportional entropy of dynamic systems. J Med Med Sci. 2013;4(8):370-81.

31. Prieto S, Rodríguez J, Correa C, Soracipa Y. Diagnosis of cervical cells based on fractal and euclidian geometrical measurements: intrinsic geometric cellular organization. BMC Medical Physics 2014;14(2):1-9.

32. Correa C, Rodríguez J, Prieto S, Álvarez L, Ospino B, Munévar A, et al. Geometric diagnosis of erythrocyte morphophysiology: geometric diagnosis of erythrocyte. J Med Med Sci. 2012;3(11):715-20.

33. Rodríguez J, Prieto S, Correa C, Bernal P, Puerta G, Vitery S, et al. Theoretical generalization of normal and sick coronary arteries with fractal dimensions and the arterial intrinsic mathematical harmony. BMC Med Phys. 2010;10:1-6.

34. Rodríguez J. Método para la predicción de la dinámica temporal de la malaria en los municipios de Colombia. Rev Panam Salud Pública. 2010;27(3):211-8.

35. Rodríguez J, Prieto S, Correa C, Pérez C, Mora J, Bravo J, et al. Predictions of CD4 lymphocytes' count in HIV patients from complete blood count. BMC Medical Physics 2013;13:3.

36. Rodríguez J, Bernal P, Prieto P, Correa C, Álvarez L, Pinilla L, et al. Predicción de unión de péptidos de Plasmodium falciparum al HLA clase II. Probabilidad, combinatoria y entropía aplicadas a las proteínas MSP-5 y MSP-6. Archivos de Alergia e Inmunología Clínica. 2013;44(1):7-14

37. Rodríguez J. Dynamical systems applied to dynamic variables of patients from the Intensive Care Unit (ICU). Physical and mathematical mortality predictions on ICU. J Med Med Sci. 2015;6(8):102-108. 